\title{
Beneficial Effect of Leptin on Spatial Learning and Memory in Streptozotocin-Induced Diabetic Rats
}

\author{
Mohsen Ghasemi ${ }^{1}$, Bamdad Zendehbad², Hoda Zabihi², Mahmoud Hosseini ${ }^{3}$, Mousa Al Reza Hadjzadeh², \\ Parichehr Hayatdavoudi ${ }^{2}$
}

\begin{abstract}
${ }^{1}$ Department of Physiology, North Khorasan University of Medical Sciences Faculty of Medicine, Bojnurd, Iran
${ }^{2}$ Department of Physiology, Neurocognitive Research Center, Mashhad University of Medical Sciences Faculty of Medicine, Mashhad, Iran ${ }^{3}$ Department of Physiology, Neurogenic Inflammation Research Center, Mashhad University of Medical Sciences Faculty of Medicine, Mashhad, Iran
\end{abstract}

Background: Diabetes mellitus is a chronic disease which may be accompanied by cognitive impairments. The expression of the obesity gene $(\mathrm{ob})$ is decreased in insulin-deficient diabetic animals and increased after the administration of insulin or leptin. Plasma leptin levels are reduced in the streptozotocin (STZ)-induced diabetic rats. Therefore, the deleterious effects of diabetes on memory may be due to the reduction of leptin.

Aims: Investigate the effect of subcutaneous injection of leptin on spatial learning and memory in STZ-induced diabetic rats.

Study Design: Animal experimentation.

Methods: The rats were divided into three groups: 1control, 2- diabetic, and 3- diabetic-leptin. Diabetes was induced in groups 2 and 3 by STZ injection $(55 \mathrm{mg} / \mathrm{kg})$ intraperitoneally (i.p). The animals received leptin (0.1 $\mathrm{mg} / \mathrm{kg}$ ) or saline subcutaneously (s.c) for 10 days before behavioral studies. Then, they were examined in the Morris water maze over 3 blocks after 3 days of the last injection of leptin.

Results: The travelled path length and time spent to reach the platform significantly increased in the diabetic group $(\mathrm{p}<0.001)$ and decreased with leptin treatment $(p<0.01 \& p<0.001$ respectively); also, a significant increase in path length and time was observed between the diabetic-leptin group and the diabetic group $(\mathrm{p}<0.01$, $\mathrm{p}<0.001$, respectively) in the probe test.

Conclusion: Leptin can exert positive effects on memory impairments in diabetic rats.

Keywords: Leptin, spatial memory, streptozotocin
Diabetes mellitus is a chronic and progressive disease with the chief manifestation of hyperglycemia. Metabolic abnormalities such as the long-lasting increase in blood glucose level, dyslipidemia, insulin resistance, hypertension and oxidative stress are common in diabetes mellitus. According to recent data, diabetes is a predisposing factor for Alzheimer disease (1), as older adults with type 2 diabetes sometimes develop cognitive impairments due to the impairment of insulin signaling and toxic accumulation of Amyloid Beta $(\mathrm{A} \beta)$ (2). The most affected parts are memory and mental processing speed, while cognitive skills such as attention, problem solving and general intelligence remain intact (3). The origin of impairment of cognition in diabetes is unclear. It has been shown that hyperinsulinemia compromises cognition $(2,3)$ and insulin resistance is the common feature in obesity and type 2 diabetes (4).

White adipose tissue secretes an adipocytokine, a $16 \mathrm{kDa}$ peptide hormone i.e. leptin (5). The obesity gene $(o b)$, which is mainly expressed by adipocytes (6), produces leptin $(5,7)$. Leptin is involved in controlling body weight and energy homeostasis, thermogenesis, feeding behavior and neuroendocrine status through specific leptin receptors which are mainly 
located in the hypothalamus. Leptin receptors are also found in the dentate gyrus and cornus ammonis 1 (CA1) regions of the hippocampus (8), the main region which plays an essential role in the spatial memory (9). There are six leptin or obesity receptor isoforms ( $o b \mathrm{R})$ from a-f (10). The long form of the brain leptin receptor $(o b \mathrm{Rb})$ is found in the hypothalamus in particular. Its expression has also been reported in pancreatic $\beta$ cells (4). Leptin and its receptors share structural and functional similarities with interleukin- 6 family of cytokines; cytokines have been reported as modulators of synaptic plasticity (8). An impairment of spatial learning and memory performance has been suggested in leptin-insensitive animals (10), while exogenous leptin promotes spatial learning and memory tasks in the Morris water maze $(8,9,11)$. Furthermore, the accumulation of $A \beta$ and dementia has been linked to the reduction of leptin $(7,12)$.

Expression of the $o b$ gene enhances after the administration of insulin or leptin and decreases in insulin-deficient diabetic animals. Furthermore, a reduced level of leptin in conditions of poor energy status is related to changes in insulin and glucose concentrations in human subjects. In humans and animals, plasma leptin level correlates with plasma insulin levels; therefore, it is likely that insulin and glucose regulate the production and secretion of leptin (5). It has also been suggested that leptin exerts an anti-diabetic effect in the streptozotocin (STZ) animal model of diabetes mellitus (4). Moreover, in untreated STZ-diabetic rats, the plasma leptin level is reduced $(4,5)$. A homozygous mutation of the $o b$ gene ( $o b / o b$ mouse), which hinders the production of leptin, is accompanied by insulin resistance and diabetes (4).

Altogether, these data suggest that leptin may enhance cognition in diabetes mellitus; therefore, we aimed, for the first time, to investigate the effects of subcutaneous injection of leptin on spatial learning and memory performance in STZinduced diabetic rats.

\section{MATERIALS AND METHODS}

In this study, adult male Wistar rats (180-250 g, 12 weeks old) were purchased from the animal laboratory of the School of Medicine and maintained under standard conditions $\left(12 / 12\right.$ hour light/dark cycle, $\left.22 \pm 2^{\circ} \mathrm{C}\right)$, with lights off at $6 \mathrm{pm}$. The animals had ad libitum access to food and water except during behavioral experiments. Recombinant leptin (Cell Guidance Systems Company; Cambridge, UK) and streptozotocin (Sigma- Aldrich Chemie GmbH; Taufkirchen, Germany) were purchased from a local company. STZ and leptin were dissolved in distilled water and further diluted with saline (13).

\section{Experimental groups}

The regional ethics committee approved all of the experimental procedures. The rats were divided into three groups including: 1- control (healthy, without any treatment, $\mathrm{N}=6$ ), 2- diabetic (received saline, $\mathrm{N}=5$ ), and 3- diabetic-leptin (received $0.1 \mathrm{mg} / \mathrm{kg}$ leptin, (11) $\mathrm{N}=5$ ).

Diabetes was induced by intraperitoneal injection of STZ ( $55 \mathrm{mg} / \mathrm{kg}$ body weight) (14) (groups $2 \& 3$ ) and confirmed by a cut-made tail blood sample after 72 hours by a glucometer (Clever Chek, Model TD-4230, TaiDoc Technology Corporation; New Taipei City, Taiwan). A serum glucose level higher than $250 \mathrm{mg} / \mathrm{dL}$ was considered diabetic.

Leptin and saline (the same volume) were injected subcutaneously from the $4^{\text {th }}$ day of STZ injection, once a day, for 10 days. Injection sites were alternatively changed (left/right shoulder and left/right hip, respectively) to reduce the pain and discomfort of the animal. Then, 3 days after the last leptin/ saline injection, behavioral studies were accomplished using the Morris water maze.

\section{Morris water maze apparatus and procedures}

The water maze was a black metal circular pool (diameter: $136 \mathrm{~cm}$, height: $100 \mathrm{~cm}$ ) which was filled with water (24$25^{\circ} \mathrm{C}$ ) up to $60 \mathrm{~cm}$. The maze had 4 imaginary equal geographical locations and release points (North, South, and West \& East). A cylindrical platform (diameter: $10 \mathrm{~cm}$, height: 58 $\mathrm{cm}$ ) was put into the water $2 \mathrm{~cm}$ below the water level in the core of the northwest quadrant. There were fixed visual signs at fixed positions around the room (i.e., computer, wall signs and other equipment). Just above the center of the maze, there was a roof connected camera for recording and sending the rat movements' data to a computer for further processing. At the beginning of each experiment, the rat was placed on a hidden platform for 20 seconds, and was then released into the water in 4 different predetermined quadrants and allowed to swim for up to 60 seconds. In each trial, the rat must find the platform and remain on it for 20 seconds. If the rat did not find the platform during this time period (using the visual clues), it was guided by the experimenter and allowed to remain on the platform for 20 seconds. Each rat had 30 seconds to dry and resting before the next trial. The experiment was based on a 3 block task over one day. Each block consisted of 4 trials. Then, the probe test was conducted; each rat had to swim for 60 seconds without the platform in the pool.

\section{Statistical analysis}

All data are expressed as mean and standard error of mean (Mean \pm SEM) using statistical package for the social sciences (SPSS) v.11.5 (SPSS Inc.; IL, USA). The time spent finding the platform and the path lengths travelled over three blocks 
for each rat were analyzed by repeated measures analysis of variance (ANOVA). The differences among groups and in the target quadrant were analyzed by one way ANOVA followed by Newman-keuls tests. The differences of the blood sugar against time and distance in each block were analyzed by two way ANOVA. $\mathrm{P}<0.05$ was considered statistically significant.

\section{RESULTS}

The blood sugar level was significantly increased in diabetic rats $(274 \pm 24.71)(\mathrm{p}<0.001)$ compared to the control rats (108.5 \pm 4.57$)$. Leptin treatment reduced the glucose level, although insignificantly, $(203.4 \pm 7.38)$ compared to the diabetic group. Two way ANOVA revealed significant differences of blood sugar versus swimming time in the first and second block in the diabetic group $(\mathrm{p}<0.05)$ and in the third block both in the diabetic and diabetic-leptin groups $(\mathrm{p}<0.05)$. Regarding the distance, the only significant difference was observed in the first block for both diabetic and diabetic-leptin groups $(\mathrm{p}<0.05)$. In the probe test, one way ANOVA revealed a significant difference for blood sugar level versus distance $(p<0.05)$.

There was a significant increment of the traveled path length and time spent to reach the platform in the diabetic group compared to the control group $(\mathrm{p}<0.001)$. Leptin reduced the path length $(\mathrm{p}<0.01)$ (Figure 1) and the time spent $(\mathrm{p}<0.001)$ (Figure 2) in the diabetic-leptin group compared to the diabetic group. No statistically significant difference was observed between the diabetic-leptin and the control group regarding the traveled path lengths and time spent. Also, there was a

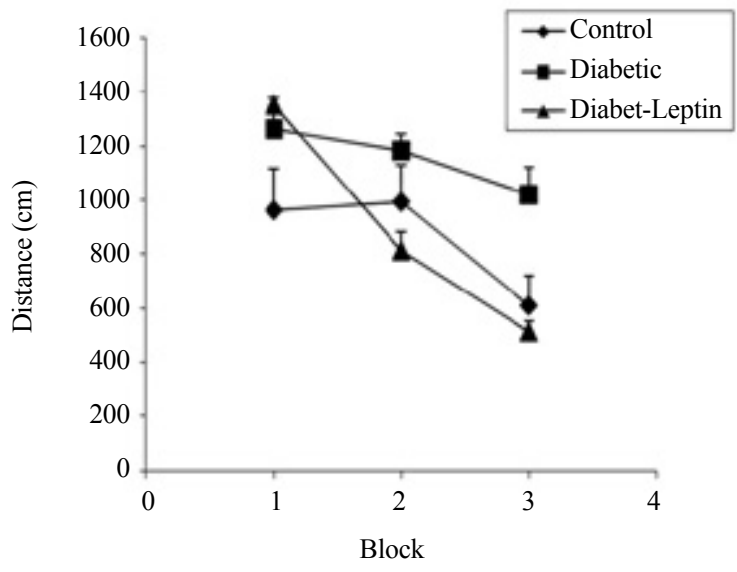

FIG. 1. Comparison of path length travelled among diabetic-leptin, diabetic and control groups. The path length increased in the diabetic group compared to the control $(p<0.001)$. Leptin significantly reduced the path length in the diabetic-leptin group compared to the diabetic group $(p<0.01)$. Data analysis was done using SPSS 11.5 by repeated measures ANOVA. $P<0.05$ was considered significant. Data are Mean \pm SEM. significant enhancement of the path length and time spent in the probe test in the diabetic-leptin group against the diabetic group ( $\mathrm{p}<0.01, \mathrm{p}<0.001$, respectively); however, no significant difference was observed between diabetic against the control group regarding the probe time and path length (Figure 3, 4).

The acquisition phase improved in the third block compared to the first block in the control group ( $\mathrm{p}<0.05)$. Diabetes impaired the acquisition phase and no significant improvement

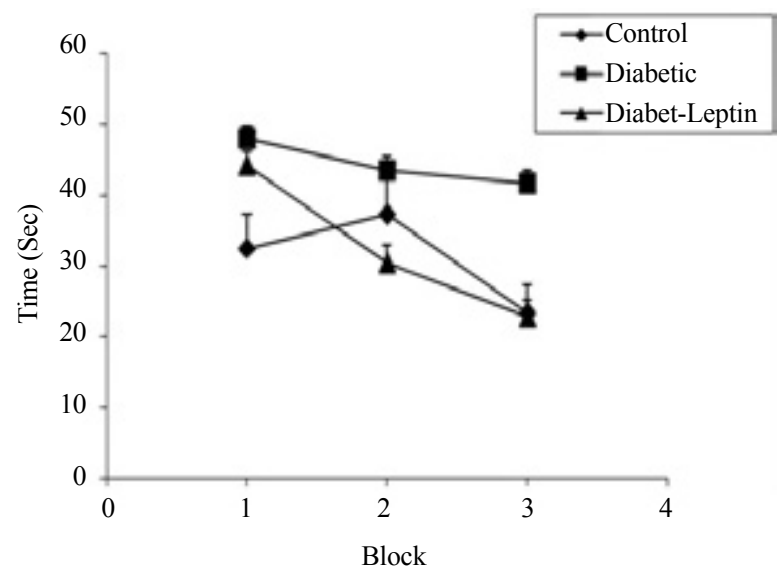

FIG. 2. Comparison of the time spent to find the platform among diabeticleptin, diabetic and control groups. The time taken to find the platform was significantly increased in the diabetic group compared to the control $(p<0$. 001). Leptin significantly reduced the time in the diabetic-leptin group compared to the diabetic group $(\mathrm{p}<0.001)$. Data analysis was done using SPSS 11.5 by repeated measures ANOVA. $P<0.05$ was considered as significant. Data are Mean \pm SEM.

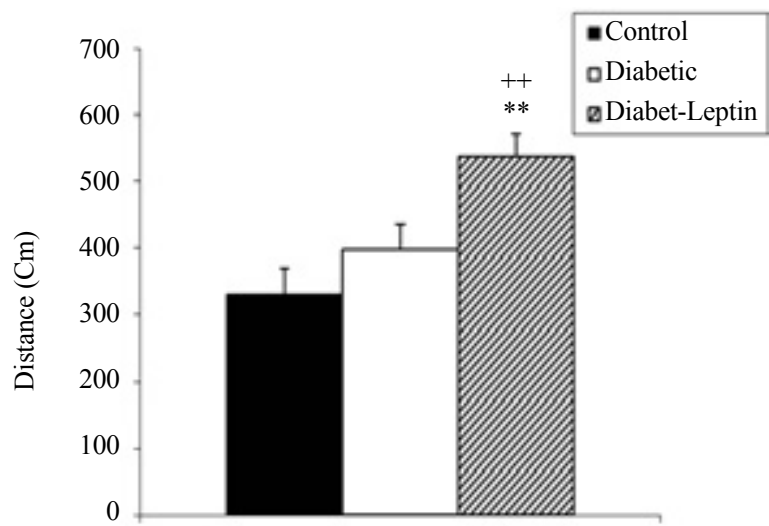

FIG. 3. Comparison of the path lengths travelled among diabetic-leptin, diabetic and control groups in the target quadrant. The traveled path lengths were not significantly different between diabetic and control groups. Leptin significantly increased the path length in the target quadrant compared to diabetic and control groups $(p<0.01)$. Data analysis was done using SPSS 11.5 by one way ANOVA; post hoc Newman-Keuls multiple comparison tests. $P<0.05$ was considered as significant. Data are Mean $\pm S E M,{ }^{* *} p<0.01$ compared with the control, $++p<0.01$ compared with the diabetic group. 


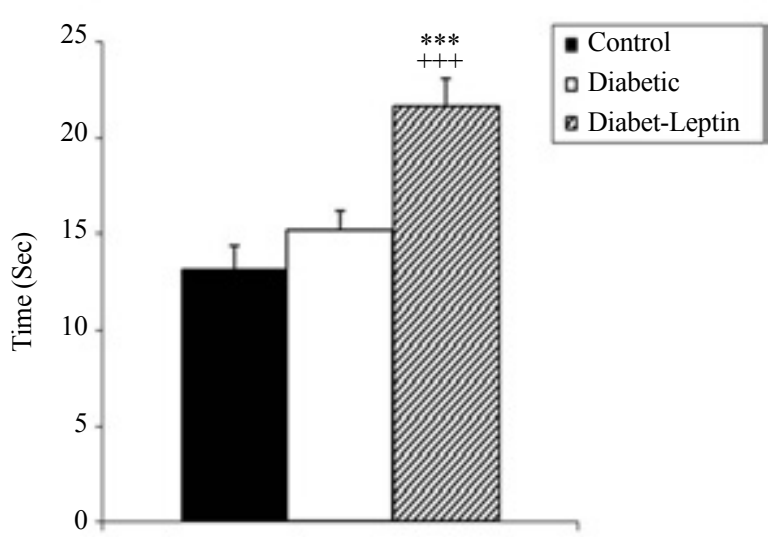

FIG. 4. Comparison of the time spent in the target quadrant among diabeticleptin, diabetic and control groups in the target quadrant. The diabetic-leptin group spent significantly more time in the target quadrant compared to both control and diabetic groups $(p<0.001)$. No significant difference was observed between the control and diabetic groups. Data analysis was done using SPSS 11.5 by one-way ANOVA; post hoc Newman-Keuls multiple comparison tests. $P<0.05$ was considered significant. Data are Mean $\pm S E M$, ${ }^{* * *} p<0.001$ compared with control, $+++p<0.001$ compared with the diabetic group.

occurred in the third block compared to the first block; furthermore, the difference between the first and second block was not significant in both diabetic and control groups. However, the leptin treatment induced a significant reduction of time and distance in the second block compared to the first block and compared to both diabetic and control groups $(\mathrm{p}<0.001)$, in addition to a significant reduction of swimming time and distance in the third block compared to the second block $(\mathrm{p}<0.001)$ and compared to the diabetic group; no difference was observed between the control and diabetic-leptin group in the third block despite the fact that in the first block, the leptindiabetic group and the diabetic group showed an almost identical increase in the swimming time and distance compared to the control group (Figure 1, 2).

\section{DISCUSSION}

According to the current study, diabetes impaired the spatial learning and memory in rats while leptin improved the memory performance in the STZ- induced diabetic rats in the Morris water maze task.

Moderate cognition impairment of learning and memory, particularly impairment of spatial learning and memory occurs in diabetic rats (15). In this study, diabetes mellitus enhanced the time and travelled paths in 3 blocks with respect to the control group, which is in agreement with similar studies of this type such as the learning deficits with severe impair- ment of Morris water maze performance following 30 days induction of STZ-induced diabetes (16), an increased escape latency and travelled path to find the hidden platform in Morris water maze test, both in 1 week and 12 weeks STZ-induced diabetes (17), the significant reduction of step down inhibitory avoidance test and an increase in acetyl cholinesterase activity after 30 day diabetes induction in rats (18), in addition to compromising cognition and behavioral aspects in two groups of juvenile rats with 10 and 20 days induction of diabetes, although the impairment progressively increased over time (19). The impairment of cognition in STZ-induced diabetic rats, which is seen as an increased escape latency and travelled distance, is in parallel to the high glucose concentration in the diabetic group in the present study. The impairment of cognition in diabetes mellitus might be due to the direct effect of STZ, high glucose level or insulin deficiency (15). On the other hand, insulin therapy and moderate glycemic control have been shown to improve the results in the Morris water maze $(4,15)$. According to the present study, the blood glucose level reduced by about $26 \%$ following leptin treatment. Therefore, it is likely that glucose reduction is the cause of the improved performance. Alterations of glucose metabolism, interactions with $\mathrm{A} \beta$ or altered expression of neuronal cell adhesion molecules (NCAMs) are among the potential mechanisms involved in spatial learning and memory deficits in diabetes mellitus $(2,16)$. Amyloid- $\beta$ accumulation in neurons can impair memory through the Janus kinase and signal transducer and activator of transcription (JAK-STAT) pathway in the hippocampus, and the functional long form of the leptin receptor ( $o b \mathrm{Rb})$, which is largely expressed in the hippocampus, is associated with the JAK2-STAT5 pathway (20). Leptin decreases A $\beta$ production in vivo and in vitro and inhibits tau phosphorylation (21) in addition to the negative effect on vascular remodeling through nitric oxide (NO)-dependent pathways (22). On the other hand, STZ reduces the leptin levels by $60 \%$ while insulin therapy enhances the leptin levels (23).

In view of the data in each block, in the acquisition phase, the third block indicates a reduction of distance travelled in the control group that is in agreement with other experiments of this type, which indicate that performance will improve over training blocks (9). In the diabetic-leptin group, the reduction in distance was greater than in the control group. Leptin has been shown to reduce the travelled path length compared to saline-administered rats (24). It has also been shown that medium doses of intraperitoneal leptin, $30 \mathrm{~min}$ before behavioral experiments, improve spatial learning and memory in the Morris water maze (11).

It is remarkable in the retention phase that there is no significant difference between the control and diabetic groups, despite significant impairment in the acquisition phase; it seems 
that whenever memory consolidation takes place, it is not vulnerable to diabetes effect, at least in a short period of time, while leptin plays a powerful effect at this stage. In the study by Oomura et al. (8), an intravenous injection of leptin improved the performance of rats in the Morris water maze test, which was accompanied by an improvement in the long-term potentiation (LTP) in the CA1 region, yet a reduced long-term depression (LTD) in the hippocampus. Moreover, the intrahippocampal injection of leptin, 30 minutes before Morris water maze, improved the performance of rats (9). Administration of leptin in the hippocampus 3 minutes after T-maze foot shock avoidance and step-down inhibitory avoidance training in mice improved the retention phase; however, leptin injection 24 hours after training did not reveal the same results (7).

It has been suggested that short-term regulation of leptin is dependent on insulin; however, in another study, leptin induced lower insulin secretion through the direct inhibition of pancreatic $\beta$-cells (4). According to some in vitro studies, leptin has a negative effect on glucose metabolism; however, in vivo observations have demonstrated that leptin has an insulin-sensitizing effect, which may be associated with the central mechanisms (25). The current study results are in agreement with insulin sensitizing effects. Regarding these facts and the results of the present study, it is suggested that the deleterious effects of diabetes on memory are at least in part due to the leptin reduction.

The other positive memory effects of leptin might include N-methyl D-aspartate (NMDA) receptor-mediated transmission and LTP (26), calcium-calmodulin dependent protein kinase II (CaMK II) signaling (27), changing of the neuronal/synaptic structure and function and promoting neuronal survival and proliferation (6) or altering the glucocorticoid expression in the hippocampus (23). Because neural stem/ progenitor cells co-express both glucocorticoid receptors (GR) and leptin receptor b, it is possible that they have interactions with each other (28) and to control hippocampal health and homeostasis (29). Leptin inhibits the detrimental effects of chronic stress and the resultant elevated levels of glucocorticoids on neurogenesis and behavior (28), in addition to positive effects on the spatial memory by reducing the anxiety (24), which may at least partly play a role in the results of the present study. Furthermore, leptin induces aromatase expression in adipose tissue followed by the elevation of estrogen (30); estrogen and its receptors are involved in learning, so the effect of leptin on memory may in part be due to its effects on estrogen (31) through the increased amounts of nitric oxide and acetylcholine in the brain (32). Leptin also triggers the release of NO in some tissues including the hypothalamus, anterior pituitary gland (33) and mesenteric vasculature (34). Since leptin receptors are also present in the hippocampus, it is possible that leptin, through NO, plays a helpful role in memory in the hippocampus. Our results are also in agreement with results indicating the positive effect of leptin on spatial learning and memory, in particular in diabetic rats. No significant difference between leptin-treated animals and the control group were observed in this study.

Briefly, the current study revealed the positive effects of leptin on spatial learning and memory in STZ-induced diabetic rats. It seems that the valuable effect of leptin on memory is a multifactorial outcome, not a single effect. The underlying mechanisms of this impact remain to be elucidated in further studies.

In conclusion, the administration of leptin at the dose of 0.1 $\mathrm{mg} / \mathrm{kg}$ has a beneficial impact on spatial learning and memory in STZ-induced diabetic rats, which at least in part may be due to the reduction of the blood glucose level.

Ethics Committee Approval: Ethics committee approval was received for this study from the ethics committee of the university (Grant number: 900891).

\section{Informed Consent: N/A.}

Peer-review: Externally peer-reviewed.

Author contributions: Concept - M.A.R.H., B.Z.B., M.G.; Design - M.H., M.A.R.H.; Supervision - M.H., M.A.R.H., B.Z.B., P.H.; Resource - P.H., M.G., M.H., H.Z.; Materials - M.G., M.A.R.H., B.Z.B.; Data Collection \&/or Processing - B.Z.B., H.Z., M.A.R.H., M.H.; Analysis \&/or Interpretation - P.H., M.H., M.A.R.H.; Literature Search - P.H., B.Z.B., M.H., H.Z.; Writing - P.H., B.Z.B., M.H., M.A.R.H., H.Z., M.G.; Critical Reviews - M.A.R.H., M.H., P.H., H.Z., M.G., B.Z.B.

Acknowledgements: The authors would like to thank Council of Research of Mashhad University of Medical Sciences and North Khorasan University of Medical Sciences for providing financial supports.

Conflict of Interest: No conflict of interest was declared by the authors.

Financial Disclosure: This study has received financial support from Mashhad University of Medical Sciences and North Khorasan University of Medical Sciences.

\section{REFERENCES}

1. Huber JD, Vangilder RL, Houser KA, Jason D, Kimberly A. Streptozotocin-induced diabetes progressively increases bloodbrain barrier permeability in specific brain regions in rats. $\mathrm{Am} \mathrm{J}$ Physiol Heart Circ Physiol 2006;291:2660-8. [CrossRef]

2. McNay EC, Recknagel AK. Brain insulin signaling: a key component of cognitive processes and a potential basis for cognitive impairment in type 2 diabetes. Neurobiol Learn Mem 2011;96:432-42. [CrossRef]

3. Ryan CM, Freed MI, Rood JA, Cobitz AR, Waterhouse BR, Strachan MW. Improving Metabolic Control Leads to Better 
Working Memory in Adults With Type 2 Diabetes. Diabetes Care 2006;29:345-51. [CrossRef]

4. Ceddia RB, Koistinen HA, Zierath JR, Sweeney G. Analysis of paradoxical observations on the association between leptin and insulin resistance. FASEB J 2002;16:1163-76. [CrossRef]

5. Havel PJ, Uriu-hare JY, Liu T, Stanhope KL, Stern JS, Keen $\mathrm{CL}$, et al. Marked and rapid decreases of circulating leptin in streptozotocin diabetic rats : reversal by insulin. Am J Physiol 1998;274:1482-91.

6. Li X, Aou S, Oomura Y, Hori N, Fukunaga K, Hori T. Impairment of Long-Term Potentiation and spatial memory in Leptin receptor-deficient rodents. Neuroscience 2002;113:607-15. [CrossRef]

7. Farr SA, Banks WA, Morley JE. Effects of leptin on memory processing. Peptides 2006;27:1420-5. [CrossRef]

8. Oomura Y, Hori N, Shiraishi T, Fukunaga K, Takeda H, Tsuji M, et al. Leptin facilitates learning and memory performance and enhances hippocampal CA1 long-term potentiation and CaMK II phosphorylation in rats. Peptides 2006;27:2738-49. [CrossRef]

9. Gisou M, Soheila R, Nasser N. Evaluation of the effect of intrahippocampal injection of leptin on spatial memory. African $J$ Pharm Pharmacol 2009;3:443-8.

10. Beccano-Kelly D, Harvey J. Leptin : A Novel Therapeutic Target in Alzheimer's Disease ? Int Journal of Alzheimers Dis 2012;2012:594137.

11. Rasi S, Mohaddes G, Naghdi N, Ilkhani MVH. Evaluation of the Effect of Peripheral Injection of Leptin on Spatial Memory. Int $J$ Neurol 2006;8:1-7.Available from www.ISPUB.COM/IJN.

12. Ding Y, Qiao A, Wang Z, Goodwin JS, Lee E, Block ML, et al. Retinoic acid attenuates beta-amyloid deposition and rescues memory deficits in an Alzheimer's disease transgenic mouse model. J Neurosci 2008;28:11622-34. [CrossRef]

13. Palou March M, Tobaruela Arbona A. Univ de les Illes Balears. Use of leptin in the treatment of alterations in food habits. Spain, EP2005967A1. 2008.

14. Bibak B, Khalili M, Rajaei Z, Soukhtanloo M, Hadjzadeh M, Hayatdavoudi P. Effects of melatonin on biochemical factors and food and water consumption in diabetic rats. Adv Biomed Res 2014;3:1-7.

15. Biessels G, Kamal A, Ramakers GM, Urban IJ, Spruijt BM, Erkelens DW, et al. Place Learning and Hippocampal Synaptic Plasticity in Streptozotocin-Induced Diabetic Rats. Diabetes 1996;45:1259-66. [CrossRef]

16. Baydas G, Nedzvetskii VS, Nerush PA. Altered expression of NCAM in hippocampus and cortex may underlie memory and learning deficits in rats with streptozotocin-induced diabetes mellitus. Life Sci 2003;73:1907-16. [CrossRef]

17. Babri S, Azami SH, Mohaddes G. Effect of Acute Administration of loganin on Spatial Memory in Diabetic Male Rats. $A d v$ Pharm Bull 2013;3:91-5.

18. Schmatz R, Mazzanti CM, Spanevello R, Stefanello N, Gutierres $\mathrm{J}$, Corrêa M, et al. Resveratrol prevents memory deficits and the increase in acetylcholinesterase activity in streptozotocin-induced diabetic rats. Eur J Pharmacol 2009;610:42-8. [CrossRef]
19. Ravishankar R, Sanjiva DK. Effects of Duration of Diabetes on Behavioural and Cognitive Parameters in Streptozotocin-Induced Juvenile Diabetic Rats. Malays J Med Sci 2011;18:26-31.

20. Marwarha G, Prasanthi JRP, Schommer J, Dasari B, Ghribi O. Molecular interplay between leptin, insulin-like growth factor-1 , and b-amyloid in organotypic slices from rabbit hippocampus. Mol Neurodegener 2011;1:1-18. [CrossRef]

21. Greco SJ, Sarkar S, Johnston JM, Tezapsidis N. Leptin regulates Tau phosphorylation and Amyloid through AMPK in Neuronal Cells. Biochem Biophys Res Commun 2009;380:98-104. [CrossRef]

22. Catal V, Rodr A, Javier G, Fortu A. Leptin Inhibits the Proliferation of Vascular Smooth Muscle Cells Induced by Angiotensin II through Nitric Oxide-Dependent Mechanisms. Mediators Inflamm 2010;2010:1-10.

23. Harvey J, Solovyova N, Irving A. Leptin and its role in hippocampal synaptic plasticity. Prog Lip Res 2006;45:369-78. [CrossRef]

24. Karakaş A, Coşkun H, Kizilkaya FU. Memory-enhancing effects of the leptin hormone in Wistar albino rats : sex and generation differences. Turk J Biol 2013;37:222-9.

25. Morrison CD. Leptin signaling in brain : A link between nutrition and cognition ? Biochim Biophys Acta 2009;1792:401-8.

26. Patel BK, Koenig JI, Kaplan LM, Hooi SC. Increase in plasma leptin and lep mRNA concentrations byfood intake is dependent on insulin. Metabolism 1998;47:603-7. [CrossRef]

27. Oomura Y, Aou S, Fukunaga K. Prandial increase of leptin in the brain activates spatial learning and memory. Pathophysiology 2010;17:119-27. [CrossRef]

28. Garza JC, Guo M, Zhang W, Lu XY. Leptin restores adult hippocampal neurogenesis suppressed by chronic unpredictable stress and reverses glucocorticoid-induced inhibition of GSK3 $\beta / \beta$ catenin signaling. Mol Psychiatry 2012;17:790-808. [CrossRef]

29. Foster TC. Role of estrogen receptor alpha and beta expression and signaling on cognitive function during aging. Hippocampus 2013;22:656-69. [CrossRef]

30. Liu E, Samad F, Mueller BM. Local adipocytes enable estrogendependent breast cancer growth Role of leptin and aromatase. Adipocyte 2013;2:165-9. [CrossRef]

31. Witty CF, Foster TC, Semple-Rowland SL, Daniel JM. Increasing Hippocampal Estrogen Receptor Alpha Levels via Viral Vectors Increases MAP Kinase Activation and Enhances Memory in Aging Rats in the Absence of Ovarian Estrogens. PLoS One 2012;7:1-10. [CrossRef]

32. Li B, Wang LU, Liu Y, Chen Y, Zhang Z, Zhang J. Jujube promotes learning and memory in a rat model by increasing estrogen levels in the blood and nitric oxide and acetylcholine levels in the brain. Exp Ther Med 2013;5:1755-9. [CrossRef]

33. Mastronardi CA, Yu WH, Mccann SM. Resting and circadian release of nitric oxide is controlled by leptin in male rats. Proc Natl Acad Sci U S A 2002;99:5721-6. [CrossRef]

34. Hacioglu A, Algin C, Pasaoglu O, Pasaoglu E, Kanbak G. Protective effect of leptin against ischemia-reperfusion injury in the rat small intestine. BMC Gastroenterol 2005;5:1-7. [CrossRef] 